\title{
Moderate Adverse Event
}

National Cancer Institute

\section{Source}

National Cancer Institute. Moderate Adverse Event. NCI Thesaurus. Code C84264.

An adverse event for which only minimal, local, or noninvasive intervention (e.g. packing, cautery) is indicated; or that limits instrumental activities of daily living (ADLs, e.g., shopping, laundry, transportation, or ability to conduct finances). 